\title{
Wandel durch Bruch?
}

\section{Mentalitätengeschichtliche Betrachtungen zum Missbrauch in der katholischen Kirche}

\author{
Hans Zollner SJ
}

\section{Verleugnung, Vertuschung, Verweigerung und die double crises}

Welle um Welle von Nachrichten über den sexuellen Missbrauch von Kindern, Jugendlichen und Schutzbefohlenen und dessen Vertuschung branden an den Kirchenmauern an. Das geht nun schon fast 40 Jahre so, denn so lange ist es her, dass in Kanada und kurz darauf in den USA über Priester berichtet wurde, die sexuelle Gewalt gegen Kinder und Jugendliche verübt haben. Besonders befördert durch die Enthüllungen des "Spotlight Team“ des Boston Globe im Jahre 2002 richtete sich die Aufmerksamkeit der Medien und der Öffentlichkeit zunächst vor allem in westlichen anglophonen Ländern auf den Missbrauch in der katholischen Kirche. Die zweite große Welle von Meldungen zu diesem Thema kam 2010 durch die Berichte über Missbrauch am CanisiusKolleg in Berlin ins Rollen und erreichte im Laufe der darauffolgenden Jahre auch jene Länder, in denen aus verschiedenen Gründen in der Öffentlichkeit nicht darüber berichtet und öffentlich gesprochen worden war.

Mit dem Bekanntwerden der Anklagen gegen den früheren Erzbischof von Washington, McCarrick sowie der Präsentation des "Grand Jury Reports“ in Pennsylvania Mitte 2018 brach eine neue Sturmflut los, diesmal nicht nur oder nicht hauptsächlich wegen der bestürzend hohen Zahlen von Missbrauchsopfern und Tätern unter den Klerikern - fast könnte man meinen, die kirchliche und nicht kirchliche Öffentlichkeit habe sich an diese monströsen Zahlen gewöhnt. Vielmehr rückten zum ersten Mal vorrangig das Versagen nicht nur eines Bischofs oder Provinzials, sondern das jahrzehntelange Verleugnen und Vertuschen von Missbrauch durch die Hierarchie und deren völlig unzulänglichen Reaktionsweisen in den Blick. Die zusätzlichen Wunden, die durch ein auf Selbstverteidigung, Schutz der Institution und völliges Verkennen der Bedürfnisse von Betroffenen ausgerichtetes System eben diesen Betroffenen und ihren Familienangehörigen zugefügt wurden, sowie das Fehlen einer Kultur der Rechenschaftspflicht im gesamten Machtgefüge der katholischen Kirche sind seither mit Wucht in den Vordergrund gerückt. Das hat z.B. in den USA dazu geführt, dass man nicht mehr nur von der abuse crisis im Blick auf den Missbrauch und die Einzeltäter spricht, sondern dass 
mittlerweile der Begriff the double crises gängig geworden ist: die Krise, die der Missbrauch selbst ausgelöst hat, und die ungeheure Vertrauenskrise, die viele Menschen in der Kirche - und zwar Laien, Ordensleute und Priester gleichermaßen - am Willen ihrer Bischöfe und Provinziäle zur wirklichen Aufarbeitung und Prävention von Missbrauch zweifeln lässt und die massive Fragen nach der Eignung der Amtsinhaber für die Bewältigung ihrer Aufgaben aufwirft. Die „Kölner Kirchenkrise“, die seit Ende 2020 zu in dieser Schärfe bisher in Deutschland nicht gekannten Schlagzeilen gegen einen Kardinal und einen weiteren Erzbischof führte, stellte allen vor Augen, dass auch in unseren Breiten die bis vor einigen Jahren scheinbar Unantastbaren angreifbar geworden sind und dass die Glaubwürdigkeit der Kirchenleitung wegen ihres insgesamt erschreckend mangelhaften Umgangs mit den Missbrauchsopfern und den Tätern weitgehend verspielt ist.

Die Wogen der Empörung, der Wut und der Verbitterung rollen unaufhaltsam, und doch scheint sich hinter den dicken Mauern der Kirchenfestung zu deren Verteidigern und Bewohnern nicht nur Kleriker, sondern auch allzu willfährige Laien gezählt werden können - nichts zu bewegen. Immer wieder werden dieselben Fehler wiederholt, immer wieder kommt es zu denselben Reaktionsmustern, immer wieder prallen die Wellen offenbar wirkungslos ab. Das führt dazu, dass mehr und mehr Menschen die Kirche entweder innerlich aufgeben oder sie amtlich verlassen, auch jene, die bisher sehr identifiziert waren mit ihrem Glauben und ihren Gemeinden, seien es Laien oder auch Kleriker. Viele haben mittlerweile den Eindruck gewonnen, dass die Institution Kirche nicht reformierbar sei, dass der Herde ihre Hirten abhandengekommen seien (und nicht umgekehrt) und dass die Kirche in der jetzigen Gestalt untergehen müsse, damit etwas Neues und Lebendiges entstehen kann. Hinzu kommt, dass seit Jahren und Jahrzehnten dieselben Forderungen nach Veränderung vorgetragen werden und nichts (oder viel $\mathrm{zu}$ wenig) an aggiornamento geschieht. Selbst die Einführung von entsprechenden Gesetzesvorgaben scheint keine Wirkung zu zeigen. Die Kirche und ihre Repräsentanten wirken wie das "Schloss“ im gleichnamigen Roman von Franz Kafka: eine Bürokratie, die undurchschaubar ist und an ihren inneren Widersprüchen erstickt; ein Schlossherr, der als Mensch im Verborgenen bleibt und wirkt, aber alles beherrscht; das Schloss selbst, zu dem man nicht vordringen kann, weil es keine einfachen und nachvollziehbaren Wege hinein gibt.

Besonders erschreckend ist dabei, dass Verleugnung und Vertuschung von Missbrauchsfällen, Versetzung von Missbrauchstätern statt ihrer Bestrafung, die Verweigerung oder Verzögerung von Aufarbeitung im Sinne einer umfassenden Bestandsaufnahme von geschehenen Verbrechen und des Umgangs mit Betroffenen und Tätern, sowie die oftmals halbherzige Vorgehensweise bei der Prävention kein lokales Phänomen sind. All dies lässt 
sich deckungsgleich überall in der katholischen Welt, das heißt in fast allen Ländern dieser Erde feststellen: eine Art von Einheit, die wahrlich nicht erstrebenswert ist.

Offensichtlich liegt das an etwas, was der katholischen Kirche tief in den Knochen steckt: einer Mentalität, oder besser: Mentalitäten, bei denen weder Zwangsmaßnahmen (Gesetzesverschärfungen) noch Bildungsmaßnahmen auf allen Ebenen ausreichen, um eine effektive Veränderung von Einstellung und Verhalten zu erzielen. Was sind diese spezifisch „katholischen“ Mentalitäten, die beim Thema Missbrauch zu so widerspenstigem und unbelehrbarem (Re-)Agieren führen, und wie kann man sie in ihrer geschichtlichen Entwicklung und Wirkmächtigkeit verstehen?

\section{Mentalitätengeschichte}

Mentalitätengeschichte ist ein geschichtswissenschaftlicher Ansatz, der in der zweiten Hälfte des 20. Jahrhunderts durch die École des Annales begründet wurde und besonders im französischen Raum Anklang fand. ${ }^{1}$ In dieser interdisziplinären Forschungsrichtung geht es um die Erforschung der Entstehung, Bedeutung und historischen Entwicklung von Mentalitäten, ${ }^{2}$ näherhin um die Frage „welchen Anteil der Mensch als denkendes, fühlendes, wünschendes Wesen an (jenen) Prozessen genommen hatte ${ }^{\text {“3 }}$, durch die Menschen zu dem wurden, was sie waren. Eine solche „Historische Anthropologie ${ }^{4}$ ist auf der Suche nach dem subjektiven Faktor in der Geschichte, „nach historischer Subjektivität, nach vergangenem Seelenleben, vergangener Sensibilität. ${ }^{5}$ Anders gesagt geht es um ein Verstehen menschlichen Selbstverständnisses sowie menschlicher Einstellungen angesichts persönlicher, regionaler oder international bedeutsamer Ereignisse oder gesellschaftlicher

1 Vgl. François Dosse, L'Histoire en miettes: des Annales à la „nouvelle histoire“. Paris 1987; Philippe Poirrier, Les enjeux de l'histoire culturelle. Paris 2004.

2 Vgl. Philippe Ariès, Die Geschichte der Mentalitäten, in: Jacques LeGoff/Roger Chartier/ Jacques Revel (Hrsg.), Die Rückeroberung des historischen Denkens. Grundlagen der Neuen Geschichtswissenschaft. Frankfurt a. M. 1994, 138-165.

3 Ulrich Raulff, Vorwort, in: ders. (Hrsg.), Mentalitäten-Geschichte. Zur historischen Rekonstruktion geistiger Prozesse. Berlin 1987, 7-17, hier 8.

4 Zur Historischen Anthropologie siehe die Zeitschrift Historische Anthropologie:Kultur, Gesellschaft, Alltag, Böhlau/Köln ab 1993; Claudia Bruns/Tilmann Walter, Von Lust und Schmerz: Eine Historische Anthropologie der Sexualität. Köln 2004; Jakob Tanner, Historische Anthropologie zur Einführung. Hamburg 2016; Aloys Winterling (Hrsg.), Historische Anthropologie. Stuttgart 2006.

5 Raulff, Vorwort, 8. 
Wirklichkeiten und umgekehrt darum, wie diese Haltungen, Entwicklungen und Geschehen prägen: Wie haben Menschen gefühlt, gedacht und ihr Handeln begründet?

Um diese affektiven, kognitiven und ethisch-handlungsorientierten Dispositionen zu ergründen, braucht es ein ,joint venture, an dem Psychologie, Kultur- und Ethnoanthropologie, Sozialgeschichte und zahlreiche andere Bindestrich-Geschichten partizipieren."6 Nach Burke richtet sich dabei das Interesse besonders auf die Beschreibung von mentalen Orientierungsmustern und kollektiven Haltungen, die sich im Denken einfacher Leute ebenso wie der formell gebildeten Eliten widerspiegeln. ${ }^{7}$ So soll herausgefunden werden, welche unausgesprochenen und oft unbewussten Annahmen und Wahrnehmungen das Alltagsdenken und die in einem bestimmten geschichtlichen Kontext angenommenen kollektiven Vorstellungen und Werte bestimmten. Dabei geht es um Inhalt und Form dieser Grundannahmen. Neben dem begrifflichen Ausdruck in Metaphern, Kategorien und Symbolen soll also auch die innere Struktur von Einstellungen und Annahmen freigelegt werden, die sich im Lauf relativ langer Zeiträume gebildet hat und die Menschen in ihrem Erleben und Handeln als Einzelne und in größeren Gruppen mehr und stärker prägen, als ihnen bewusst ist.

Die Themen und Methoden der Mentalitätengeschichte sind dementsprechend breit gestreut: das Spektrum reicht von mikrohistorischen Untersuchungen von Dörfern oder Regionen über die Analyse von bildlichen und schriftlichen Zeugnissen sowie alltäglichen Lebensweisen bis hin zu Bildern oder persönlichen Quellen wie Tagebüchern oder Briefen. Ein wirkmächtiges Beispiel für den Ansatz und die Methodenvielfalt der Mentalitätengeschichte ist Philippe Ariès' „Geschichte der Kindheit“, in der er die Entwicklung der Vorstellungen und Wahrnehmungen von Kindheit in den westeuropäischen Gesellschaften seit dem Mittelalter erörtert. ${ }^{8}$

Mentalitätengeschichte fragt also nach „kollektiven Weltbildern, Einstellungen, nach alltagsweltlich verankerten Orientierungsmustern, die das Handeln der Menschen bestimmen und ihre Haltung in konkreten Situationen

6 Ebd., 9 .

7 Vgl. Peter Burke, Varieties of Cultural History. Ithaca/NY 1997.

8 Original: Philippe Ariès, L'Enfant et la vie familiale sous l'Ancien Régime. Paris 196o. Deutsche Erstausgabe: Geschichte der Kindheit. München 1975. Vgl. die Rezeption von Ariès bei Hubertus Lutterbach, Kinder und Christentum: Kulturgeschichtliche Perspektiven auf Schutz, Bildung und Partizipation von Kindern zwischen Antike und Gegenwart. Stuttgart 2010. 
bestimmen ${ }^{\prime 9}$, die das Entstehen, das Aufrechterhalten und die Weitergabe sozialer Strukturen und ihre Anwendung im individuellen Leben maßgeblich beeinflussen. Es wird angenommen, dass diese Prozesse vielschichtig sind, sich wechselseitig verstärken und zum großen Teil unbewusst ablaufen. Das erklärt auch, warum solche tief und lange verankerten Mentalitäten sich in der Regel nur langsam ändern bzw. sich von außen nur schwer verändern lassen. Wenn man Mentalitätengeschichte im Bewusstsein dieser Eigenart und Begrenztheit betrachtet, dann kann sie neue Themenfelder erschließen und Einsichten jenseits einliniger kognitiver Erklärungsmuster eröffnen.

Für die Bestandsaufnahme dessen, was in den letzten Jahrzehnten - vielleicht sollte man sagen Jahrhunderten - in der katholischen Kirche hinsichtlich Missbrauch und seiner Vertuschung geschehen ist, bietet ein mentalitätengeschichtlicher Blick zumindest einen wichtigen ergänzenden Ansatzpunkt für die Beantwortung von drei Fragen, die mir im Lauf der Jahre aus der Begegnung mit Menschen aus allen Kontinenten erwachsen sind:1) Wie kann es sein, dass überall auf der Welt in katholischen Kreisen (beim Leitungspersonal und im Kirchenvolk) die gleichen emotionalen Reaktionsmuster, kognitiven Äußerungen und Verhaltensweisen festgestellt werden können, wenn es um das Thema Missbrauch geht? 2) Warum ist der Lernfortschritt insgesamt so gering, obwohl in weiten Teilen der Weltkirche beachtliche Anstrengungen unternommen wurden und werden, um sowohl das Wissen über Risikofaktoren und die Konsequenzen von Missbrauch zu erweitern, als auch um die rechtlichen Grundlagen zu schaffen, um Verantwortungsübernahme und Rechenschaftspflicht zu definieren und umzusetzen? 3) Warum gibt es eine, für viele Außenstehende, aber auch mehr und mehr für Kircheninsider rational nicht nachvollziehbare Abwehr, sich mit diesem Thema auseinanderzusetzen obwohl man wissen könnte, dass gerade das Nicht-Auseinandersetzen zu seiner Perpetuierung beitragen wird? Damit hängt eng zusammen die Frage, warum man nicht von dem lernt, was die Kirche durch den Missbrauchsskandal in Ländern wie den USA, Irland oder Australien schon unter großen Verlusten an Glaubwürdigkeit, Energie oder auch Geld erlebt hat.

Es drängt sich der Eindruck auf, dass es eine spezifisch katholische Mentalität gibt, die es schwer macht, aktiv auf Betroffene zuzugehen und entstandenes Leid anzuerkennen, Schuld zu bekennen, Aufarbeitung zu leisten und sich für Prävention zu engagieren. Stattdessen lassen sich weltweit sehr ähnliche Reaktionsmuster erkennen: Betroffene werden abgewiesen und abgewertet;

9 Rudolf Schlögl, Mentalitätengeschichte, einzusehen unter https://www.uni-konstanz.de/ FuF/Philo/Geschichte/Tutorium/Themenkomplexe/Grundlagen/Forschungsrichtungen/ Mentalitatengeschichte/mentalitatengeschichte.html, zuletzt aufgerufen am 31.1.2021. 
Schuld und Verantwortung werden geleugnet, bagatellisiert oder abgeschoben; Aufarbeitung wird entweder verweigert oder an Anwälte, Kirchenrechtler, Psychologen und Psychiater delegiert; Präventionsarbeit wird Spezialisten übertragen, ohne dass sie in das normale kirchliche Handeln integriert worden wäre.

Wie konnte es so weit kommen bei einer Institution, die das Evangelium der Liebe Gottes und der Nächstenliebe verkünden soll und deren Gründer freiwillig sein Leben hingab, anstatt sich selbst mit Macht, Geld oder spitzfindigen Ausflüchten zu retten?

\section{Katholische Mentalitätselemente - woher kommen sie und wie wirken sie?}

Angesichts der Komplexität und schieren Breite des Themas können die folgenden Ausführungen nicht mehr als eine holzschnittartige Auflistung dessen bieten, was in die bestimmende Mentalität in der katholischen Kirche gegenüber dem Missbrauch mit einfließt. Schon hier sei vermerkt, was weiter unten wieder aufgegriffen werden wird: Natürlich kann man in der ältesten existierenden und größten Institution der Welt nicht alle und alles über einen Kamm scheren. Natürlich bräuchte es mehr Daten und differenzierende Darstellungen. Dennoch kann man es meines Erachtens wagen, Elemente zu benennen, die so etwas wie eine katholische Mentalität im Angesicht des Missbrauchs ausmachen.

Viele dieser Elemente wurden aufgrund wichtiger Veränderungsprozesse in Kirche und Gesellschaft im Laufe der vergangenen etwa 250 Jahre für die Kirche von heute prägend. Die zu vertiefende Ausgangsthese lautet: Die katholische Kirche, die in Europa bis zu Beginn der Neuzeit im Religiösen unumschränkte und im Politischen weitreichende Macht hatte, hat seit der Reformation und dann verstärkt seit der Mitte des 18. Jahrhunderts ihre Vorrangstellung auf verschiedenen Feldern eingebüßt und ist in eine Defensivposition geraten. In Kultur und Wissenschaften kam es mehr und mehr zu einer Emanzipation von der kirchlichen Bevormundung und zu einer zunehmenden Frontstellung von aufgeklärter Philosophie, Naturwissenschaften und Humanwissenschaften (wie wir das heute nennen) gegen kirchliche Positionen. Besonders einschneidend war dabei die Auseinandersetzung mit den Idealen der Französischen Revolution, der Evolutionstheorie und der modernen Psychologie. Die Säkularisation und das Ende des Heiligen Römischen Reiches Deutscher Nation, das Entstehen der Nationalstaaten und die Einverleibung des Kirchenstaates in das Königreich Italien wurden als traumatischer Angriff 
auf die Souveränität und weltliche Macht der katholischen Kirche erlebt und gedeutet. Die Industrialisierung und die Abwanderung großer Bevölkerungsteile in die Städte erschwerte den unmittelbaren Kontakt der Priester mit den Gläubigen deutlich.

Im 2o.Jahrhundert agierten die nationalistischen wie auch kommunistischen Bewegungen und Regime explizit kirchenfeindlich. Das Leid der Weltkriege und der vielen anderen Kriege sowie die unermesslichen Gräuel des Holocaust, von Genoziden und unzähligen Menschenrechtsverletzungen brachten viele Menschen nicht nur zum Verzweifeln, sondern auch zum Zweifeln an der christlichen Gottesbotschaft. Frauen haben in vielen weltlichen Bereichen und Institutionen vorher unerreichbare Rollen und Positionen übernommen, in der katholischen Kirche geht dieser Prozess je nach Wahrnehmung sehr langsam bis gar nicht voran. Die in den letzten Jahrzehnten des 20. Jahrhunderts einsetzende und sich rasant weiterentwickelnde Digitalisierung und die damit im wahrsten Sinne des Wortes grenzenlose Pluralisierung stellen eine grundlegende Herausforderung für die herkömmlichen kirchlichen Kommunikations-, Gebets- und Ritualformen dar. Auch wenn Papst Franziskus mit seinen Schriften und Reden eine unbestritten wichtige Stimme im Kampf gegen den Klimawandel und für die Bewahrung und den Erhalt der Schöpfung ist, so spielt dies für die meisten Zeitgenossen eher eine untergeordnete Rolle bei dem, was viele als das wichtigste Menschheitsthema schlechthin betrachten. Bei den großen Fragen der Sexual- und Lebensethik - etwa in der Auseinandersetzung um die Abtreibungs- und Euthanasiegesetze sowie in der Genderdiskussion - wird die Kirche fast unweigerlich als die intransigente Nein-Sagerin wahrgenommen, die kaum konkrete und konstruktive Debattenbeiträge macht. Und sehr viele hören nicht (mehr) hin mit dem Argument, die Kirche habe wegen der Unmenge von Missbrauchsfällen ihre moralische Autorität völlig verspielt.

Diese wenigen Stichworte sollen genügen, um deutlich zu machen, dass die Institution katholische Kirche speziell in den letzten 250 Jahren die Entwicklungen in zentralen Lebensbereichen als eine politische und weltanschauliche Entmachtung und als einen Angriff auf ihre Eigenständigkeit erlebt hat. Aus tiefenpsychologischer Sicht könnte man das mit einer fortdauernden und fortschreitenden narzisstischen Kränkung bzw. Verwundung vergleichen. Als weitreichende Folgen dieses Erlebens können bei vielen in der Kirche eine latent oder prononciert pessimistische Einstellung gegenüber der "Welt" angenommen werden, ein trotziges Abwehrverhalten und der Versuch, jede Art von Wandel in der Liturgie, in der Glaubenslehre und im äußeren Erscheinungsbild aufhalten bzw. die Zeit zurückdrehen zu wollen. Während das Selbstbild lange so erlebt werden konnte: „Wir sind allmächtig, 
und wir können alles erklären und steuern, weil wir unfehlbar sind“ (von Pius IX. im längsten Pontifikat der Kirchengeschichte auf den Gipfel getrieben), empfinden viele in der Kirchenleitung in den letzten Jahrzehnten mehr und mehr so: „Wir sind den kritischen Medien und anderen Stimmen ausgeliefert, wir werden ungerecht behandelt" und jüngst auch "Wir werden überfordert" (z.B. der Magdeburger Bischof Feige). ${ }^{10}$

Erschwerend kommt hinzu, dass das Verhältnis von Glaube und Vernunft, von Kirche bzw. Religion und Welt (betrachtet als „Moderne “11, „Post-Moderne“ oder „Post-Post-Moderne“) nicht wirklich geklärt wurde, auch nicht durch das Zweite Vatikanische Konzil, das diesbezüglich zwei Schritte vor und einen zurück machte. ${ }^{12}$ Kardinal Carlo Maria Martini SJ beschrieb drei Wochen vor seinem Tod im Jahr 2012 die Situation der Kirche so: „Die Kirche ist 200 Jahre zu spät dran. Warum schüttelt sie sich nicht? Haben wir Angst?"13 Angst, Verzagtheit und Mutlosigkeit sprechen augenscheinlich aus den Äußerungen vieler Kirchenführer, die sich in ihre Wagenburg zurückziehen, sich weitgehend isolieren und gegen jede Art von Kritik immunisieren. Bezeichnenderweise wird der Widerspruch zwischen dem Gestus solcher Botschaften (der von den Insignien der göttlichen und weltlichen Macht früherer Zeiten begleitet wird) einerseits und ihrer tatsächlichen Wirkung andererseits von Mal zu Mal größer: Oft wirkt dies wie bei des „Kaisers neuen Kleidern“ (vgl. das Kunstmärchen von H. C. Andersen), wo der Kaiser es „aushält“(!) und die Prozession

10 Vgl. Hubert Wolf, Der Unfehlbare: Pius IX. und die Erfindung des Katholizismus im 19. Jahrhundert. München 2020; Peter Neuner, Der lange Schatten des I. Vatikanums: Wie das Konzil die Kirche noch heute blockiert. Freiburg/Basel/Wien 2019; Vorwürfe und Forderungen. Bischof Feige sieht Bischöfe mit immer mehr Kritik konfrontiert, 3.12.2020, einzusehen unter https://www.domradio.de/themen/bistuemer/202O-12-03/vorwuerfeund-forderungen-bischof-feige-sieht-bischoefe-mit-immer-mehr-kritik-konfrontiert, zuletzt aufgerufen am 31.1.2021. Vgl. Kardinal Roger Michael Mahonys Stellungnahme vom 4.2.2013 zu den Anschuldigungen von Vertuschung und anderem Fehlverhalten gegenüber Missbrauchstätern: „Nothing in my own background or education equipped me to deal with this grave problem", einzusehen unter www.catholicculture.org/news/ headlines/index.cfm?storyid=16968, zuletzt aufgerufen am 31.1.2021.

11 Vgl. Paul Gilbert (Hrsg.), L'Uomo moderno e la Chiesa. Roma 2012.

12 Vgl. eine ausführliche Darstellung hierzu in Vittorio Conti, II „Chi della storia“. Un possibile rilancio della proposta antropologica interdisciplinare di L. M. Rulla in dialogo con la psicologia fenomenologica di G. Arciero. Mailand 2018.

13 „La Chiesa è rimasta indietro di 200 anni. Come mai non si scuote? Abbiamo paura?", einzusehen unter https://www.corriere.it/cronache/12_settembre_o2/le-parole-ultimaintervista_cdb2993e-f5ob-11e1-9f3o-3eeo1883d8dd.shtml, zuletzt aufgerufen am 31.1.2021. 
weiterlaufen lässt, obwohl er erkennt, dass die Leute sein Nacktsein bemerkt haben. ${ }^{14}$

Kernsätze dieser Wagenburg-Mentalität, einer trotzigen Selbstbehauptung und derSelbststilisierung zu Opfern(!), die ich im Rahmen meiner Vortragstätigkeit als Präsident des Centre for Child Protection der Universität Gregoriana von Klerikern und Nicht-Klerikern aus allen Teilen der Welt so oder ähnlich gehört habe, sind: „Ich bin der Bischof (Provinzial etc.), ich brauche mich nicht zu rechtfertigen vor jenen, die die Besonderheit der Kirche nicht verstehen. "Die Medien wollen die Kirche zerstören - das ist ein klares Zeichen, dass wir auf dem Weg der Nachfolge des Gekreuzigten sind. Wir werden angegriffen, weil wir unbequem sind.“ „Den Opfern geht es nur um's Geld.“ „Bei den anderen Religionen, im Sport, in der Schule, in den Familien - gibt es viel mehr Missbrauch, und keiner schaut hin.“ „Wir müssen zusammenhalten. Mein Priester ist Teil der Familie.“ "Er hat mir versprochen, er werde es nicht mehr tun. Fehler macht jeder. Wer bereut und sich zu bessern verspricht, dem muss verziehen werden.“ "Ich habe ein reines Gewissen, ich kann das mit meinem Herrgott klären.“ „Ich brauche keine Kontrolle und keine Fortbildung, ich weiß, was ich tue."

Dies und noch mehr könnte man unter dem Schlagwort „Klerikalismus" subsumieren: eine Mentalität also, die sich allein aus dem Innehaben eines priesterlichen oder bischöflichen Amtes ableitet, sich gegenüber dem gemeinen Kirchenvolk - inklusive Laien im kirchlichen Dienst - als höherwertig zu fühlen und Ansprüche auf besondere Rechte geltend machen zu können. ${ }^{15}$ Wenn die Verbindung von Gemeindeleitung und sakramentalem Dienst dazu führt, dass der Priester und mehr noch der Bischof allzuständig ist und man sich mehr und mehr allmächtig fühlt, dann ist die Gefahr groß, dass man über kurz oder lang der Versuchung erliegt, diese Macht für sich auszunutzen bzw. andere für die eigenen Zwecke zu missbrauchen. Viele Betroffene von Missbrauch sagen, dass sie die sexualisierte Gewalt vor allem als

14 Hans Christian Andersen, Sämmtliche Märchen, 1862. Des Kaisers neue Kleider, einzusehen unter https://maerchen.com/andersen/des-kaisers-neue-kleider.php, zuletzt aufgerufen am 31.1.2021: „Aber er hat ja nichts an!' rief zuletzt das ganze Volk. Das ergriff den Kaiser, denn es schien ihm, sie hätten Recht; aber er dachte bei sich: ,Nun muss ich die Prozession aushalten.' Und die Kammerherren gingen noch straffer und trugen die Schleppe, die gar nicht da war".

15 Diese „implizite Amtstheologie“ konnte lange mit einer „habituellen Vertrauensseligkeit“ nicht nur auf Seiten von Betroffenen von Missbrauch rechnen: vgl. Regina Heyder/ Ute Leimgruber, Spiritueller und sexueller Missbrauch an erwachsenen Frauen. Was aus den Berichten von Betroffenen zu lernen ist, in: Barbara Haslbeck u.a. (Hrsg.), Erzählen als Widerstand: Berichte über spirituellen und sexuellen Missbrauch an erwachsenen Frauen in der katholischen Kirche. Münster 2020, 187-220, hier 197. 
Machtmissbrauch erlebt haben und drücken das oft mit diesen oder ähnlichen Worten aus: „Der sexuelle Akt war schlimm, schmerzhaft, schamhaft. Das, was mich am meisten aber verletzt und getroffen hat, war, dass ich nicht ausweichen konnte, dass der, der mich missbraucht hat, übermächtig war. ${ }^{16}$ Wer sich selbst als hervorgehoben und unantastbar versteht, und wem von anderen eine besondere Ehrerbietung und absolutes Vertrauen entgegengebracht wird, bei dem kann sich leicht eine klerikalistische Mentalität einstellen: „Weil ich Priester bin, deshalb darf ich mir nehmen, was mir passt. Nicht, weil ich Gott suchen und Christus folgen will, nicht, weil ich mehr reflektiert hätte, nicht, weil ich mehr über den Glauben nachgedacht habe, nein, sondern schlicht, weil ich Priester bin, allein deshalb steht mir das zu“.17

Darin kommen narzisstische Persönlichkeitselemente zum Ausdruck, die durch die vorherrschende Art der Ausbildung noch befördert werden können - in Priesterseminaren, in denen die Seminaristen oft in einer Sonderwelt leben, die weder mit derjenigen ihrer Altersgenossen Ähnlichkeit hat, noch auf die wirkliche Lebenssituation von Priestern in Pfarrgemeinden heute vorbereitet. In dieser, in verschiedener Hinsicht abgesicherten Umgebung können Beziehungsmuster gedeihen, in denen gesunde Kritikfähigkeit praktisch verunmöglicht wird, man voneinander abhängig werden kann und weitreichende und langanhaltende Seilschaften entstehen können. $\mathrm{Zu}$ welchen dysfunktionalen Abläufen dies innerhalb einer „männerbündischen“ Eigenwelt führen kann, hat zuletzt der McCarrick-Report vor Augen geführt. Drei der vier Bischöfe aus New Jersey, die im Jahr 2000 vom damaligen Nuntius gebeten wurden, darüber Auskunft zu geben, ob die Anschuldigungen gegen

16 Vgl. die Erläuterung der Kampagne „Sprechen hilft“ der Unabhängigen Beauftragten zur Aufarbeitung des sexuellen Kindesmissbrauchs: „Mit dem Leitsatz ,Wer das Schweigen bricht, bricht die Macht der Täter' ruft die Kampagne Betroffene dazu auf, über den Missbrauch zu sprechen und sich damit von der Macht der Täter bzw. Täterin - versinnbildlicht durch eine übermächtige Hand, die den Mund zuhält - zu befreien." Abschlussbericht der Unabhängigen Beauftragten zur Aufarbeitung des sexuellen Kindesmissbrauchs, Dr. Christine Bergmann, April 2011, einzusehen unter https://beauftragter-missbrauch. de/fileadmin/Content/pdf/Presse_Service/Publikationen/Abschlussbericht-derUnabhaengigen-Beauftragten-zur-Aufarbeitung-des-sexuellen-Kindesmissbrauchs.pdf, 30, zuletzt aufgerufen am 31.1.2021.

17 Hier sei nur angedeutet, dass ein solches priesterliches (Selbst- und oft auch Fremd-) Bild letztlich auf einer tendenziell monophysitischen Christologie aufruht, die die Wirklichkeit und Bedeutung der menschlichen Natur Jesu und die damit gegebenen Grenzen verleugnet - mit allen erwartbaren Konsequenzen für eine „nicht-inkarnierte“ Spiritualität. Vgl. Hans Zollner, „Brannte uns nicht das Herz?“ Aspekte des Priesterseins, in: Geist und Leben 82, 2009, 161-166; ders., In Gott verwurzelt - eingetaucht in das Herz der Welt. Sendung und Auftrag der Kirche zu Beginn des 21. Jahrhunderts, in: Zeitschrift für katholische Theologie 135, 2013, 194-211. 
McCarrick wegen sexueller Handlungen mit jungen Männern wahr waren, "provided inaccurate and incomplete information to the Holy See“. ${ }^{18}$ Dies bestätigt die Einschätzung, dass die Ernennung zum Bischof oder die Untersuchung des Fehlverhaltens eines Bischofs nicht nur in den Händen von Bischöfen liegen sollte, sondern dass darin auch unabhängige Experten einbezogen werden sollten.

Für jene, die in der Kirche groß geworden sind, die ihr alles verdanken Ausbildung, Rolle, Ansehen - und die auch deshalb ihre Aufgabe darin sehen, die Institution zu schützen, ist es mitunter schlicht unvorstellbar, dass in der Kirche und durch Kirchenvertreter das Leben von Wehrlosen und Schutzbefohlenen zerstört wurde. Denn wer könnte in den Spiegel schauen und dort eine schreckliche Fratze entdecken, ohne zu erschrecken und sofort wegzuschauen? Mächtige Verdrängungsmechanismen kommen ins Spiel, die dazu führen, dass das Leid Betroffener und ihrer Familienangehörigen geleugnet oder bewusst ausgeblendet wird - wie jüngst von dem früheren Aachener Bischof Mussinghoff bezeugt -, und dass die bleibenden Risiken bei Tätern spiritualisiert und nicht in ihrer Schwere erkannt werden. ${ }^{19}$

Dass diese Mentalität gerade bei jenen zu finden ist, die dazu beauftragt sind, sich um das Seelenheil der Menschen zu sorgen, ist zutiefst alarmierend und zerstört nicht selten die Grundlagen der Gottesbeziehung von Betroffenen. In der Tat ist daher für viele von ihnen sowie für Sekundärbetroffene (z.B. Familienangehörige) der Verlust des Vertrauens und des Glaubens die tiefste Wunde, die der Missbrauch hinterlässt. Diese spirituelle Dimension des Missbrauchs wurde und wird in der Kirche weitgehend ausgeblendet im Gegensatz zu rechtlichen und psychologischen Fragen. Allzu oft ging und geht es vor allem darum, einen öffentlichen Skandal zu vermeiden. Eine realitätsferne Idealisierung der Institution und eine große Scham, Fehlverhalten oder Verbrechen öffentlich zuzugeben, verstärken diese Tendenz. Non fare brutta figura oder „Du sollst keinen Skandal produzieren“ wird als elftes kirchliches Gebot zur impliziten und oft auch expliziten Handlungsmaxime, die als Vogel-Strauß-Politik oder Salami-Taktik (man gibt nur das zu, was eh schon bekannt ist) weiterhin von vielen kirchlichen Stellen praktiziert

18 Vgl. Report on the Holy See's Institutional knowledge and Decision-Making Related to Former Cardinal Theodore Edgar McCarrick (1930 to 2017), Vatikanstadt, 10.11.2020, einzusehen unter https://www.vatican.va/resources/resources_rapporto-card-mccarrick_ 20201110_en.pdf, 7, zuletzt aufgerufen am 31.1.2021.

19 Vgl. Bischof Mussinghoff: Fühlte mich mit Opfergesprächen überfordert, 9.11.2020, einzusehen unter https://www.kirche-und-leben.de/artikel/bischof-mussinghoff-fuehltemich-mit-opfergespraechen-ueberfordert, zuletzt aufgerufen am 22.2.2021. 
wird.$^{20}$ Solch ein Vorgehen kreiert fast unweigerlich einen längeren und weitreichenderen Skandal, wie sich an vielen Beispielen belegen ließe. Viele sehen eine Welle der Empörung auf die Kirche zukommen, ohne dass sie diese mit den Mitteln, die sie haben, brechen könnten. Es entsteht der Eindruck, man gehe unter. Das führt zu einer institutionellen Verunsicherung, zur Flucht vor Verantwortung, zu Lähmung und zu Fragmentierung der Erinnerung. Archivunterlagen werden zu Gunsten der Institution "frisiert" - und dies bei einer Institution, deren moralische Autorität auf ihrer Glaubwürdigkeit und Wahrhaftigkeit beruht. Doch offenbar dominiert in einer Mentalität der Selbstimmunisierung die Furcht vor der eigenen Verletzlichkeit, vor Mit-Leid, vor dem Eingeständnis von Verbrechen und Sünde so sehr, dass das Handeln dem eigenen Auftrag diametral widerspricht. Das ist umso erstaunlicher, als den einzelnen Katholiken im Sakrament der Versöhnung - der Beichte - die Vergebung Gottes zugesprochen wird unter der Bedingung, dass sie bereuen, bekennen und Schaden wiedergutmachen. Wenn die Wirkkraft des Sakramentes auch bezogen auf die Kirche als Ganzes vorausgesetzt wird, muss man fragen: Wo gab es mit Blick auf den Missbrauch Zeichen tiefer Reue, ein klares Bekenntnis zur Schuld und eine ausreichende Wiedergutmachung? Erst wenn alle drei Elemente gegeben sind, kann auch im Kontext von Missbrauch von Vergebung gesprochen werden.

Dass diese Mentalität so flächendeckend und wirkmächtig ist, liegt wohl vor allem auch daran, dass bei der Auswahl und Ausbildung der Priesterkandidaten sowie bei der Bestellung von Bischöfen und anderen Führungspersönlichkeiten die Frage der emotionalen, psychosexuellen und relationalen Reife de facto keine zentrale Rolle spielt. Das kann fatale Folgen haben, weil so jene zwei Grundbedürfnisse des Menschen nicht angesprochen und bearbeitet werden, die bei der sexuellen Gewalt zusammenwirken: Macht und Sexualität. Sexualität ist weit mehr als nur der sexuelle Akt. In der Weise, wie jemand seine/ihre Sexualität lebt, kommt seine/ihre Persönlichkeit zum Ausdruck. Sie dient vielfach dazu, auch andere tiefsitzende Bedürfnisse zu befriedigen - wie etwa nach Anerkennung, Nähe und Zuwendung, aber auch nach Bestätigung und Dominanz. Daher wirkt es besonders verheerend, wenn sich diese auch in sexuellem Begehren und Agieren oft verborgenen und unbewussten

20 Jüngstes Beispiel ist die Medienpolitik der Erzdiözese Köln mit Blick auf das nicht veröffentlichte Gutachten einer Münchener Kanzlei zu Missbrauchsfällen im Erzbistum und zu den Verantwortungsträgern im Untersuchungszeitraum. Matthias Drobinski/Christian Wernicke, Lesen und Schweigen, 6.1.2021, einzusehen unter https://www.sueddeutsche. de/medien/katholische-kirche-missbrauch-kardinal-woelki-stillschweigen-erzbistumkoeln-journalisten-1.5166778, zuletzt aufgerufen am 22.2.2021. 
Bedürfnisse nicht nur mit Machtstreben, sondern mit einer scheinbar unangreifbaren Machtposition verbinden.

Sexualität war und ist für viele Katholiken ein schambehaftetes und schwieriges Thema. Diese Feststellung ist trotz aller lehramtlichen und theologischen Äußerungen, die Sexualität als ein göttliches Geschenk darstellen und von ihrer Schönheit sprechen, seit langer Zeit zum Allgemeingut geworden. Die vielfältigen und komplexen Gründe dafür müssten in einer eigenen Untersuchung dargelegt werden. Hier sei nur auf zwei Faktoren hingewiesen, die die Mentalität in der katholischen Kirche in Bezug auf Sexualität beeinflusst haben: erstens, die neutestamentliche Naherwartung der Wiederkehr des Herrn, weswegen alles andere - auch Sexualität, Ehe und Kinder als nachgeordnet dargestellt werden; zweitens, in der lateinischen Tradition der Einfluss des heiligen Augustinus auf die Lehre von der Erbsünde, vom Lustempfinden als Sünde, und vom Geschlechtsverkehr, der nur in der Ehe zwischen Mann und Frau und nur mit dem Ziel der Fortpflanzung erlaubt ist. Die offiziellen kirchlichen Positionen etwa zu Empfängnisverhütung und Ehescheidung werden aber schon seit geraumer Zeit selbst von den meisten Katholiken kaum mehr verstanden und akzeptiert. ${ }^{21}$ In kaum einem anderen Lebensbereich erscheint die Kirche so weit vom Lebensgefühl und vom Verhalten der meisten Menschen entfernt, wie beim Thema Sexualität. ${ }^{22}$ Als besonders empörend wird es dann empfunden, wenn jene, die sexuelle Enthaltsamkeit einschärfen, selbst Minderjährige oder schutzbefohlene Erwachsene missbrauchen. Wenn dann noch diese sexuellen Verbrechen von den Kirchenoberen entweder gar nicht, zu langsam oder unverhältnismäßig nachsichtig bestraft werden, schlägt das dem Fass den Boden komplett aus und zerstört die Glaubwürdigkeit von Kirche.

Spott, Hohn und Zorn sind als Reaktionen auf diese Doppelmoral - Wasser predigen und Wein trinken - vorprogrammiert. Das betrifft vor allem auch die Frage der Bewertung von Homosexualität vor dem Hintergrund, dass

21 "The gulf between teaching and practice is wide, the teachings themselves are in dispute or ignored by Catholics, the questions cut deep, and Catholic laity have a great deal to offer from our own experience of life and love." Lisa Fullam, The Catholic Church needs a sex talk, 11.3.2013, einzusehen unter https://www.washingtonpost.com/ national/on-faith/the-catholic-church-needs-a-sex-talk/2013/o3/11/9abd3b8a-8a8c-11e28d72-dc76641cb8d4_story.html, zuletzt aufgerufen am 31.1.2021.

22 Vgl. Eberhard Schockenhoff (Hrsg.), Liebe, Sexualität und Partnerschaft: Die Lebensformen der Intimität im Wandel. Freiburg i. Br. u.a. 2019; Regina Ammicht Quinn (Hrsg.), „Guter" Sex: Moral, Moderne und die Katholische Kirche. Paderborn u.a. 2013, darin die Artikel von Theresia Heimerl und Walter Schaupp; Birgit Aschmann/Wilhelm Damberg (Hrsg.): Liebe und tu, was du willst? Die „Pillenenzyklika“ Humanae vitae von 1968 und ihre Folgen. Paderborn 2021; Eberhard Schockenhoff (posthum), Die Kunst zu lieben: Unterwegs zu einer neuen Sexualethik. Freiburg/Basel/Wien 2021. 
alle bekannten Statistiken belegen, dass die meisten sexuellen Übergriffe von Priestern gegen männliche Minderjährige gerichtet sind. Dabei ist zu bedenken, dass homosexuelle Übergriffe nicht immer auf eine eindeutig homosexuelle Orientierung schließen lassen. Zumindest in der Vergangenheit war es zum Beispiel so, dass Priester kaum direkten Kontakt zu Mädchen hatten. Ministranten waren männlich, in den Schulen unterrichteten Priester meist nur Jungen, und auch die Jugendarbeit geschah nach Geschlechtern getrennt. Die federführenden Forscherinnen der John-Jay-Studien ${ }^{23}$ aus den USA nennen die Missbrauchstäter der 1950er bis 1980er Jahre in der Kirche „Opportunisten“24: Sie nahmen sich, was sie bekamen. Das eigentliche Problem bei sexuellem Missbrauch ist nicht die sexuelle Orientierung, sondern der Missbrauch von Macht und die mangelhafte Auseinandersetzung mit grundlegenden menschlichen Bedürfnissen.

Als das Treffen der Vorsitzenden der Bischofskonferenzen und der Generaloberen und der Generaloberinnen im Februar 2019 vorbereitet wurde, war klar, dass kritisch auf die strukturellen, institutionellen Komponenten geschaut werden muss, die den Missbrauch und seine Vertuschung ermöglicht haben. Damit rückte ein weiteres Element in den Blick, das in der katholischen Mentalität eine große Rolle spielt: die bemerkenswerte Scheu vor Verantwortungsübernahme. Der Eindruck drängt sich auf, dass kirchliche Führungspersonen zwar die Ehren und das Gefühl von (im jeweiligen Aufgabenbereich bisher praktisch unbegrenzter) Macht für erstrebenswert halten. Doch wenn es darum geht, ähnlich wie in Politik oder Wirtschaft, Verantwortung zu übernehmen und persönliche Konsequenzen bis hin zu einem Rücktritt zu ziehen, dann fehlt fast immer der Mut zum eigenen Schritt. Als Begründung wird dann oft vorgeschoben, dass man von Gott in dieses Amt berufen worden sei und deshalb auch treu bleiben müsse, oder dass man die Entscheidung den römischen Behörden oder dem Papst überlasse. Ein

23 The Nature and Scope of Sexual Abuse of Minors by Catholic Priests and Deacons in the United States 1950-2002. A Research Study Conducted by the John Jay College of Criminal Justice, Februar 2004, einzusehen unter www.usccb.org/sites/default/files/ issues-and-action/child-and-youth-protection/upload/The-Nature-and-Scope-of-SexualAbuse-of-Minors-by-Catholic-Priests-and-Deacons-in-the-United-States-1950-2002. pdf, zuletzt aufgerufen am 31.1.2021; The Causes and Context of Sexual Abuse of Minors by Catholic Priests in the United States, 1950-2010. A Report Presented to the United States Conference of Catholic Bishops by the John Jay College Research Team, Mai 2011, einzusehen unter https://www.usccb.org/sites/default/files/issues-and-action/childand-youth-protection/upload/The-Causes-and-Context-of-Sexual-Abuse-of-Minors-byCatholic-Priests-in-the-United-States-1950-2010.pdf, zuletzt aufgerufen am 31.1.2021.

24 Karen J. Terry, Stained Glass: The Nature and Scope of Child Sexual Abuse in the Catholic Church, in: Criminal Justice and Behavior 35(5), 2008, 549-569, hier 567. 
probates Mittel für mehr Transparenz und Klarheit in der Definition von Verantwortlichkeit wird im protestantisch geprägten angelsächsischen Raum mit accountability zum Ausdruck gebracht - zu Deutsch: Rechenschaftspflicht. Man kann accountability ins Italienische, Spanische, Französische oder Portugiesische übersetzen, indem man umschreibt, was gemeint ist. Es gibt aber in keiner dieser vier Sprachen, die in Ländern gesprochen werden, in denen die meisten Katholiken leben, ein äquivalentes Substantiv, das „Rechenschaftspflicht" im hier gebrauchten Sinn wiedergeben könnte. Wenn man für etwas keinen Begriff hat, dann bedeutet das, dass man nicht darüber nachdenkt, spricht und es entsprechend thematisiert. Ein kleines Beispiel, dass dies ganz offensichtlich bei accountability in den katholischen Ländern der Fall ist, ist die vatikanische Informationspolitik, die - mit wenigen Ausnahmen wie dem McCarrick-Bericht - die Gründe für außerordentliche Bischofsrücktritte nicht offenlegt.

Im Rahmen eines solchen Artikels soll dies genügen, um einige Elemente dessen zu benennen, woraus sich die spezifisch katholische Mentalität angesichts des Missbrauchs und seiner Vertuschung zusammensetzt. Weiter oben wurde ebenfalls darauf hingewiesen, dass dies natürlich nicht für alle im gleichen Maße zutrifft. Doch zumindest Spurenelemente davon werden sich bewusst und unbewusst bei den allermeisten wiederfinden lassen, die sich der spirituellen und kulturellen Wirkmacht der katholischen Kirche zurechnen und in ihr eine führende Aufgabe innehaben.

\section{Paradoxien und die Möglichkeit eines Mentalitätswandels}

Angst, Verunsicherung, Scham im Umgang mit Sexualität oder Machtstreben sind bei jedem und jeder in unterschiedlichem Maße ausgeprägt. Doch es gibt auch einige Beobachtungen, die die Gesamtsituation nicht einfach schwarz und weiß erscheinen lassen. Manches daran erscheint paradox und provoziert zum Weiterdenken. Im weiteren Sinn gehören auch diese Paradoxien zur katholischen Mentalität.

Am offensichtlichsten ist, dass es in der einen Kirche gleichzeitig Betroffene und Täter gibt. Wenn man den Statistiken über Missbrauch durch Kleriker glauben darf, dann ist zu vermuten, dass relativ viele Betroffene - vor allem jene, die mit niemandem darüber sprechen - die Kirche nicht verlassen haben. Das heißt auch, dass das Trauma, das die Betroffenen erlebt haben, in ihnen und durch sie auch in der Kirche gegenwärtig ist: wenn sie an Gottesdiensten teilnehmen, wenn sie sich in Pfarreien oder bei der Caritas engagieren, wenn sie geistlichen Rat suchen. Dies sollte niemals vergessen werden: Bei der 
Feier der Liturgien, in Gemeindeversammlungen, bei Zusammenkünften und Diskussionen braucht es daher immer große Sensibilität. Dass kaum einmal für Betroffene von sexueller Gewalt in der Kirche gebetet wird, spricht Bände darüber, wie sehr sie - ihr Schmerz, ihre Verbitterung, ihr Suchen und ihre Hoffnung - vom Glaubensalltag abgespalten werden. Anstatt mit ihnen gemeinsam einen Weg zu suchen, auf dem sie ihre eigenen spirituellen Suchbewegungen und ihre persönlichen und professionellen Kompetenzen artikulieren können, wird vielen nach eigener Beschreibung die Tür der Pfarrei, der Ordensprovinz oder der Diözese vor der Nase zugeschlagen.

Auch mit Blick auf die andere Seite - die Täter - ist festzuhalten, dass kaum wahrgenommen wird, dass viele von ihnen - selbst nach einer etwaigen Entlassung aus dem Klerikerstand - Mitglieder der Kirche waren und sind. Das müsste zu denken und zu tun geben: Wie behandelt man Menschen in den eigenen Reihen, die Verbrechen verübt haben, wie jene, die sich dessen bewusst sind, wie auch jene, die es nicht wahrhaben können? Wie kann man begründen, dass Täter Aufsicht und Begleitung brauchen, gerade auch, um weiteren Missbrauch durch sie zu verhindern?

In der einen Kirche gibt es unter jenen, die mit der Strafverfolgung beauftragt sind, solche, die dies gewissenhaft tun, und es gibt solche, die vertuschen, verleugnen, verharmlosen. Letztere könnte man als „Sekundärtäter“ bezeichnen, deren rechtspositivistisches, abwertendes und hartherziges Verhalten Betroffene und Sekundärbetroffene nach deren Zeugnis manchmal mehr verletzt als die eigentliche Missbrauchstat.

Mehr und mehr Menschen der einen Kirche verstehen, wie wichtig das Safeguarding ist, und engagieren sich dafür. Andere hingegen meinen, man solle nicht so viel über dieses Thema reden, damit man sich wieder auf die „eigentlichen pastoralen Fragen“ konzentrieren könne. Die Kirche in ihren Mitgliedern ist also in der Terminologie der Traumatherapeutin Ursula Enders gleichzeitig eine traumatisierende und eine traumatisierte Institution. ${ }^{25}$ Diese Spannung ist für Menschen auf beiden Seiten des Spektrums schwer auszuhalten.

Ein weiteres Paradox wurde oben schon angedeutet: Klerikalismus gibt es nicht nur bei Klerikern. Auch bei Laien sind die gleichen Phänomene zu finden: Wenn man sich nämlich aus einer bestimmten kirchlichen Position heraus bestimmte Freiheiten nimmt und ungerechtfertigte Ansprüche

25 Ursula Enders, Traumatisierte Institutionen. Wenn eine Einrichtung zum Tatort sexueller Ausbeutung durch einen Mitarbeiter/eine Mitarbeiterin wurde, 2004, einzusehen unter https://www.zartbitter.de/o/Eltern_und_Fachleute/6o3o_traumatisierte_institutionen. pdf, zuletzt aufgerufen am 31.1.2021. 
aufgrund der eigenen Rolle auf bestimmte Räumlichkeiten, Ausstattung, Dienstauto, Prestige etc. erhebt. Allerdings kommt bei geweihten Klerikern explizit die geistlich-religiöse Dimension zur Begründung der Unantastbarkeit und Besonderheit hinzu.

Wenn man nach dem Kirchenbild fragt, dann haben viele Menschen die Vorstellung einer absolutistischen, zentral durchstrukturierten, klar organisierten und autoritär-hierarchischen Institution. Zweifellos gab und gibt es solche Vorgehensweisen. Aber meist sind dies entweder Maßnahmen von römischen Behörden, etwa Lehrbeanstandungsverfahren, bei denen man nicht weiß, wer was und warum entschieden hat. Oder ein Pfarrer benimmt sich wie ein Bischof in seiner Pfarrei, und ein Bischof in seinem Bistum wie der Papst, ohne Widerspruch oder Kritik zu dulden. Letzteres macht ein für viele überraschendes Phänomen deutlich: Die katholische Kirche offenbart auf verschiedenen Ebenen und in vielen Abläufen ein kaum zu durchschauendes Konglomerat von Zuständigkeiten und verwickelten Befehls- und Verantwortungsketten. Gerade diese Unklarheit begünstigt Missbrauch und seine Vertuschung, wie schon 2010 im Deetman-Report unmissverständlich beschrieben wurde: Man schiebt sich die Bälle hin und her, am Ende ist niemand schuld. ${ }^{26}$ Ein Beispiel: Wenn ein Ordenspriester, der Pfarrer in einer Diözese ist, einen Jugendlichen in einer anderen Diözese missbraucht: Welcher Obere ist dann für was zuständig? Wie soll da ein Betroffener wissen, an wen er sich wenden soll? Für viele ist unverständlich, dass zwar von Rom oder der Bischofskonferenz Gesetze und Leitlinien verabschiedet werden, dass diese aber von denjenigen nicht richtig gekannt bzw. zur Kenntnis genommen und allzu oft nicht eingehalten werden, die für ihre Ausführung vor Ort die Verantwortung tragen. ${ }^{27}$ Erschwerend kommt hinzu, dass das kirchliche Strafrecht hinsichtlich seiner Auslegung und seiner Anwendung mit dem staatlichen Strafrecht kaum zu

26 Wim Deetman, Seksueel Misbruik van Minderjarigen in de Rooms-Katholieke Kerk. Amsterdam 2011, einzusehen unter https://www.rkkerk.nl/wp-content/uploads/2016/12/ Seksueel-misbruik-minderjarigen-RKK_Deetman-deel-1.pdf, zuletzt aufgerufen am 31.1.2021.

27 Das betrifft z.B. auch die offiziellen Dokumente zur Priesterausbildung seit „Pastores dabo vobis“" (von Johannes Paul II. aus dem Jahr 1992), dessen Betonung der zentralen Bedeutung der „menschlichen“ Ausbildungseinheiten (psycho-sexuelle und emotionale Reife, Beziehungsfähigkeit usw.) in den Leitlinien der Kleruskongregation von 2016 („Das Geschenk der Berufung zum Priestertum - Ratio Fundamentalis Institutionis Sacerdotalis“) eins zu eins übernommen wurde. Doch in den Priesterseminaren und der Fortbildung für Priester spielt dieser Bereich bei Weitem nicht die Rolle, der ihm laut höchster zuständiger Autorität in der Kirche zukommen sollte. Vgl. Swarna Anna Mary Thumma, Human formation in the training to the priesthood: with specific attention to psychosexual integration, Estratto di dissertazione. Rom 2020. 
vergleichen ist: Es fehlen z.B. klare und publizierte Kriterien der Vergleichbarkeit, die Definition elementarer Prozessrechte, eine Gewaltenteilung nach staatlichem Vorbild. Insgesamt hat dies das Vertrauen in die kirchliche Gerichtsbarkeit sehr beschädigt und den Eindruck befördert, dass Suprema Lex nicht salus animarum ist, wie es im Kanon $175^{2}$ des CIC heißt, sondern dass es letztlich darum geht, die eigenen Leute zu schützen.

In der Öffentlichkeit wird weiterhin das Bild des allmächtigen Pfarrers kolportiert, der auf dem Dorf mehr zu sagen hat als der Bürgermeister. Das mag es in einigen Weltgegenden noch geben, und in Einzelfällen auch in unseren Breiten. Das Empfinden vieler Priester ist jedoch ein anderes, gerade auch wegen der Missbrauchsskandale: Sie sind zutiefst verunsichert und fühlen sich einem Generalverdacht ausgesetzt: „Bist du auch so einer von denen, die Kinder vergewaltigen?" Der beste Ausdruck für diesen Generalverdacht ist der englische Begriff für Priester, die nicht missbraucht haben: sie sind die nonoffending priests. Schon aus der negativen Definition wird klar, dass man als Priester immer mit im "Missbrauchs-Boot“ sitzt. Vor diesem Hintergrund stellen sich viele Fragen: Wie können diejenigen unterstützt und begleitet werden, die zu Unrecht allgemeinen Verdächtigungen ausgesetzt sind? Wie können Kirchenleitungen ihrer Fürsorgepflicht gegenüber ihren Mitarbeitern gerecht werden, ohne die Sensibilität für Fehlverhalten zu verlieren? Wie müsste eine Theologie des Priesterseins im Zusammenhang mit einer Theologie der Verwundbarkeit und der richtig verstandenen Machtausübung ausschauen?

Wenn diese Elemente und Paradoxien einer katholischen Mentalität Realität sind, dann kann man leicht verstehen, warum die Stimmung unter Katholiken vielerorts gedrückt, verzagt und verwirrt ist. Wenn man dann noch bedenkt, wie schwer es ist, Mentalitäten zu ändern, stellt sich die Frage, ob und wie genau das gelingen könnte. Einer der Kritikpunkte am Ansatz der Mentalitätsgeschichte ist ja gerade, dass sie schwer erklären kann, wie es angesichts der Langlebigkeit, dem Wirkungsvermögen und der Widerstandskraft von Mentalitäten gelingen kann, dass sich ebendiese verändern. Jedoch gibt es nicht nur Mikroanpassungen, die unmerklich und sehr langsam Haltungen und Einstellungen verändern, sondern auch große Krisen, die Anlass für Veränderungen sein können: „Sometimes a problem intrudes from outside and causes a crisis. Sometimes it is the crisis itself and an honest acknowledgment of it as such which enables one to ask the right questions in order to begin deepening our understanding of the issues involved and a way forward. Sometimes horizons cannot be broadened unless they are first broken. ${ }^{\text {“28 }}$

28 Cory Hayes, Even Doctors of the Church Make Theological Mistakes Now and Again, 17.9.2020, einzusehen unter https://churchlifejournal.nd.edu/articles/even- 
In der Kirchengeschichte gab es immer wieder Zusammenbrüche, die von den Zeitgenossen wohl kaum weniger dramatisch erlebt wurden als heutige double crises. Der Zug der katholischen Kirche besonders in den „alten“ katholischen Ländern rast schon seit vielen Jahren mit hoher Geschwindigkeit in Richtung einer Mauer. Mehr und mehr Menschen auch in der Kirche begreifen, dass ein echter Bruch kommen wird oder, wie es ein deutscher Bischof schon vor einigen Jahren im privaten Gespräch ausdrückte: „Alles muss zusammenbrechen, bevor es wieder neues Leben gibt."

Wie die sehr schmerzhaften und enttäuschenden Erfahrungen der vergangenen Jahre lehren, kann die Kirche das verlorengegangene Vertrauen nur dann zurückgewinnen, wenn deren Repräsentanten offen und ehrlich ihre Fehler, Verbrechen und Sünden eingestehen und alles nur irgend Mögliche tun, damit die mit Mühsal Beladenen in ihr Orte der Heilung und, wo möglich, Versöhnung finden. Dazu gehört, dass sich Kirchenführung und Kirchenvolk dem stellen, was in der Vergangenheit an Verbrechen und an Vertuschung geschehen ist, und dass von Missbrauch Betroffene an Aufarbeitungsprozessen ihren selbstverständlichen und selbstverantworteten Anteil haben. In diesem Prozess müssen die Abspaltungen bzw. Polarisierungen überwunden werden: Es braucht klarere Gesetze und Normen und eine Veränderung der Einstellung und Haltung; Psychologie und (Kirchen-)Recht und die Theologie im Angesicht des Missbrauchs sind wichtig; nur das Miteinander von Amtsträger und „Laien“ kann die Kirche auf den richtigen Weg bringen; die Zusammenarbeit von Diözesen und Orden ist für ein konsistentes und koordiniertes Vorgehen notwendig. Das Verdrängte, Verborgene und Verheimlichte an Scham, Schmerz, Angst und Mutlosigkeit muss ans Tageslicht kommen. Theologie, Psychologie, Kirchenrecht, Spiritualität müssen dabei zusammenwirken. Das ist desillusionierend und wird oft als schmerzhaft und unerträglich empfunden. Nicht wenige zerbrechen daran, andere laufen davor weg. Das ist menschlich gesehen verständlich, auch wenn es geistlich gesehen gerade die Bewährung des Glaubens an den gerechten und barmherzigen Gott wäre, der in Jesus das Leid und die Verursachung des Leides auf sich genommen hat. Jesus Christus jenseits der eingespielten und allzu oft leer erscheinenden kirchlichen Routine neu zu entdecken, gerade dort, wo Menschen durch Vertreter der Kirche unsäglich viel gelitten haben, das ist die zentrale Herausforderung an eine Kirche, die weder von Tsunami-Wellen weggerissen werden will, noch sich verschanzt in einer hermetisch abgeriegelten und sterilen Burg. Wenn die Umstände (der Gesellschaft wie diejenigen des einzelnen Lebens) sich ändern - und sie haben

doctors-of-the-church-make-theological-mistakes-now-and-again/?s=O3, zuletzt aufgerufen am 31.1.2021. 
sich nach allgemeinem Empfinden umfassend geändert -, dann muss sich auch die Weise ändern, wie wir Gott suchen und Kirche sind. Dann wird sich die katholische Mentalität wandeln. Dann wird das Wasser der Wellen nicht nutzlos anbranden, sondern die Erde tränken und Frucht tragen lassen. 